\title{
No AGN evidence in NGC 1614 from deep radio VLBI observations
}

\author{
Rubén Herrero-Illana, ${ }^{1 \star}$ Antxon Alberdi, ${ }^{2}$ Miguel Ángel Pérez-Torres, ${ }^{2,3}$ \\ Almudena Alonso-Herrero, ${ }^{4}$ Daniel González-Millán ${ }^{2}$ and Miguel Pereira-Santaella ${ }^{5}$ \\ ${ }^{1}$ European Southern Observatory (ESO), Alonso de Córdova 3107, Vitacura, Casilla 19001, Santiago de Chile, Chile \\ ${ }^{2}$ Instituto de Astrofísica de Andalucía (IAA-CSIC), Glorieta de la Astronomía s/n, E-18008 Granada, Spain \\ ${ }^{3}$ Visiting Scientist: Departamento de Física Teórica, Facultad de Ciencias, Universidad de Zaragoza, E-50009, Zaragoza, Spain \\ ${ }^{4}$ Centro de Astrobiología, CSIC-INTA,ESAC Campus, Villanueva de la Cañada, E-28692 Madrid, Spain \\ ${ }^{5}$ Department of Physics, University of Oxford, Keble Road, Oxford OX1 3RH, UK
}

Accepted 2017 May 25. Received 2017 May 19; in original form 2017 April 7

\begin{abstract}
We present deep dual-band 5.0- and 8.4-GHz European VLBI Network (EVN) observations of NGC 1614, a local luminous infrared galaxy with a powerful circumnuclear starburst ring, and whose nuclear engine origin is still controversial. We aim at detecting and characterizing compact radio structures both in the nuclear region and in the circumnuclear ring. We do not find any compact source in the central $200 \mathrm{pc}$ region, setting a very tight $5 \sigma$ upper limit of $3.7 \times 10^{36}$ and $5.8 \times 10^{36} \mathrm{erg} \mathrm{s}^{-1}$, at 5.0 and $8.4 \mathrm{GHz}$, respectively. However, we report a clear detection at both frequencies of a compact structure in the circumnuclear ring, $190 \mathrm{pc}$ to the north of the nucleus, whose luminosity and spectral index are compatible with a core-collapse supernova, giving support to the high star formation rate in the ring. Our result favours the pure starburst scenario, even for the nucleus of NGC 1614, and shows the importance of radio VLBI (very long baseline interferometry) observations when dealing with the obscured environments of dusty galaxies.
\end{abstract}

Key words: supernovae: general - galaxies: individual: NGC 1614-galaxies: nucleigalaxies: starburst.

\section{INTRODUCTION}

Galaxies with infrared (IR) luminosities higher than $10^{11} \mathrm{~L} \odot$ [luminous infrared galaxies (LIRGs)] are known to dominate the star formation (SF) at redshift $z \sim 1-2$ (e.g. Magnelli et al. 2009). The study of the physics of these objects is thus essential to understand the SF history of the Universe. Moreover, most local LIRGs contain an active galactic nucleus (AGN), which generally does not dominate their bolometric luminosity (Alonso-Herrero et al. 2012). Very long baseline interferometry (VLBI) observations provide a unique tool to study in detail the nearby sources that display similar properties to those dominating the SF activity at $z \sim 1-2$, i.e. local LIRGs. Indeed, since LIRGs are heavily enshrouded in dust, VLBI observations are among the few tools that can probe deep into the innermost pc-size regions of LIRGs, thus allowing us to pinpoint the precise location of the putative AGN as well as determining the contribution of the starburst and the AGN. For example, Pérez-Torres et al. (2010) used contemporaneous European VLBI Network (EVN) observations at 1.6 and $5.0 \mathrm{GHz}$ to discover a lowluminosity AGN (LLAGN) in Arp 299 surrounded by dozens of supernovae ( $\mathrm{SNe}$ ) and $\mathrm{SN}$ remnants.
NGC 1614 is a LIRG at a distance of 64 Mpc with an IR luminosity of $4 \times 10^{11} \mathrm{~L} \odot$, and in a late stage of a merging process. The central kpc region of NGC 1614 hosts a prominent circumnuclear ring of SF of $\sim 600 \mathrm{pc}$ diameter, revealed in $\mathrm{Pa} \alpha$ (Alonso-Herrero et al. 2001).

In Herrero-Illana et al. (2014), we used multiwavelength observations aimed at characterizing the circumnuclear region, finding an average star formation rate of $58 \mathrm{M}_{\odot} \mathrm{yr}^{-1}$ and a core-collapse SN rate of $0.4 \mathrm{SN} \mathrm{yr}^{-1}$, obtained through spectral energy distribution (SED) modelling. We also disentangled the radio thermal free-free and non-thermal synchrotron emission to determine the age of the starburst, finding an age differentiation in the ring, with the starburst in the south-east regions being younger $(\lesssim 5.5 \mathrm{Myr})$ than that in the north-west ( $\simeq 8 \mathrm{Myr}$ ).

It is widely accepted that NGC 1614 is mainly powered by a starburst. Several recent millimetre studies have been performed to determine the properties of the fuel of the SF, i.e. the molecular gas, and its inflows and outflows (Olsson et al. 2010; Imanishi \& Nakanishi 2013; García-Burillo et al. 2015; Xu et al. 2015; König et al. 2016; Saito et al. 2016, 2017). However, while the bulk of observational evidence favours a pure starburst scenario for NGC 1614 , the existence of an intrinsically weak AGN at its very centre has not yet been completely ruled out (Risaliti et al. 2000; Wilson et al. 2008; Pereira-Santaella et al. 2015b). 
Table 1. Observations summary. The codes for the participating stations are as follows (name, diameter and country): Ef: Effelsberg, $100 \mathrm{~m}, \mathrm{Germany} ; \mathrm{Wb}$ : Westerbork, 25 m, Netherlands; O6: Onsala, 20 m, Sweden; Mc: Medicina, 32 m, Italy; Nt: Noto, 32 m, Italy; Sv: Svetloe, 32 m, Russia; Zc: Zelenchukskaya, 32 m, Russia; Hh: Hartebeesthoek, 26 m, South Africa; Ys: Yebes, 40 m, Spain; Jb: Jodrell Bank, 76 m, UK; O8: Onsala, 25 m, Sweden; and Tr: Torun, 32 m, Poland. The maximum recoverable scale (MRS) is obtained as $0.6 \lambda / B_{\min }$, where $B_{\min }$ is the shortest baseline of the array.

\begin{tabular}{|c|c|c|c|c|c|c|c|c|}
\hline Code & Band & Date & EVN participating stations & $\begin{array}{l}t_{\mathrm{on}-\text { source }} \\
(\min )\end{array}$ & $\begin{array}{l}\text { FWHM } \\
\text { (mas) }\end{array}$ & $\begin{array}{l}\text { PA } \\
\left({ }^{\circ}\right)\end{array}$ & $\begin{array}{l}\text { MRS } \\
\text { (mas) }\end{array}$ & $\begin{array}{c}\mathrm{rms} \\
\left(\mu \mathrm{Jy} \text { beam }^{-1}\right)\end{array}$ \\
\hline EH030A & $X$ & $2015-05-29$ & Ef, Wb, O6, Mc, Nt, Sv, Zc, Hh, Ys & 198 & $4.0 \times 1.9$ & -14.6 & 17 & 28 \\
\hline
\end{tabular}

In this Letter, we present dual-frequency EVN radio observations of the complete circumnuclear region aimed at obtaining direct evidence or a tight upper limit to the luminosity of any putative AGN, as well as to detect and characterize any other compact radio structure across the circumnuclear ring.

\section{OBSERVATIONS AND DATA REDUCTION}

We observed NGC 1614 with EVN at $5.0 \mathrm{GHz}$ ( $C$ band) and $8.4 \mathrm{GHz}$ ( $X$ band), under project EH030 (PI: Herrero-Illana) in 2015 May/June. The observations were contemporaneous with the aim of detecting any possible compact source and characterizing its spectral shape. We used a bandwidth of $128 \mathrm{MHz}$ for both setups and a data recording of $1024 \mathrm{Mbps}$ with 2-bit sampling. We observed 3C454.3 for fringe-fitting and bandpass purposes, and J0427-0700 for phase calibration. In Table 1, we present a summary of the observations.

For data reduction, we used the NRAO AIPS (Astronomical Image Processing System) software. The process consisted of careful visual inspection and editing, amplitude calibration through system temperatures and standard phase calibration. We derived amplitude and phase gain corrections via self-calibration on the phasereference source, which were later interpolated to the target source. Imaging was also performed on AIPS, using the CLEAN algorithm (task IMAGR), with a Briggs weighting using a ROBUST parameter of 0.5 , resulting in synthesized beams of $4.7 \times 2.0$ mas $^{2}(1.61$ $\left.\times 0.87 \mathrm{pc}^{2}\right)$ and $4.0 \times 1.9 \mathrm{mas}^{2}\left(1.24 \times 0.59 \mathrm{pc}^{2}\right)$ and $\mathrm{rms}$ of 30 and $28 \mu \mathrm{Jy}$ beam $^{-1}$ for the $C$ and $X$ bands, respectively. No selfcalibration was applied. We have estimated a 5 per cent uncertainty in the flux density calibration.

While our main goal was to study the innermost region in search for a putative AGN, we imaged a larger field of view ( $\left.4 \operatorname{arcsec}^{2}\right)$ so as to detect possible compact structures in all the circumnuclear ring of the galaxy.

\section{RESULTS AND DISCUSSION}

In Fig. 1, we show our Very Large Array (VLA) image of the circumnuclear ring of NGC 1614 with overlaid regions (for the formal definition of the regions, see table 2 from Herrero-Illana et al. 2014), together with the EVN observations presented in this study of the innermost nuclear region $(\mathrm{N})$. We do not find any emission in that region above $5 \times \mathrm{rms}$ at any of the observed frequencies. The implications of these non-detections are developed in Section 3.1.

There is, however, one significant detection above $5 \times \mathrm{rms}$ in our imaged $4 \operatorname{arcsec}^{2}$ region. It corresponds to a compact source in region A (see Herrero-Illana et al. 2014), which is clear at both the $X$ and $C$ bands. We show the corresponding images in Fig. 2, while the nature of this source is discussed in Section 3.2.

\subsection{The elusive nuclear engine of NGC 1614}

The non-detection of any nuclear compact radio structure in our deep EVN observations imposes a tight limit to the radio luminosity of a putative AGN. Olsson et al. (2010) found an unresolved component $(<0.2 \operatorname{arcsec})$ in the centre of the ring at $5 \mathrm{GHz}$ using MERLIN. However, that emission is resolved out in our higher angular resolution data, pointing to a non-compact SF origin, in agreement with their interpretation.

We have established a $5 \sigma$ luminosity $\left(\nu L_{v}\right)$ upper limit for an AGN located in the nuclear region of NGC 1614 of $3.7 \times$ $10^{36} \mathrm{erg} \mathrm{s}^{-1}$ in the $C$ band and $5.8 \times 10^{36} \mathrm{erg} \mathrm{s}^{-1}$ in the $X$ band, ruling out a luminous AGN, with typical radio luminosities of $\sim 10^{42} \mathrm{erg} \mathrm{s}^{-1}$ (see e.g. Sikora, Stawarz \& Lasota 2007). Assuming a flat spectral index between 5.0 and $1.4 \mathrm{GHz}$, and a far-IR-to-radio correlation (Condon 1992) with no dispersion and the same for the AGN and for the overall emission ( $q=2.46$; Herrero-Illana et al. 2014), we derive an AGN contribution of $\lesssim 0.1$ per cent relative to the total IR luminosity.

However, the possibility of a LLAGN still remains. While most LLAGNs have detected radio cores with luminosities above our threshold (Nagar et al. 2002), there are several compact detections of very nearby galaxies in the low-luminosity end of LLAGNs that have significantly lower luminosities (e.g. NGC 4051, with $v L_{v}=$ $10^{35} \mathrm{erg} \mathrm{s}^{-1}$ at $5 \mathrm{GHz}$; Giroletti \& Panessa 2009).

Pereira-Santaella et al. (2015b) found that the mid-IR properties of the $150 \mathrm{pc}$ nuclear region of NGC 1614 are at odds with those from the starburst ring. Interestingly, the $11.3 \mu \mathrm{m}$ polycyclic aromatic hydrocarbon equivalent width and the mid-IR continuum are similar to those found in Seyfert galaxies, proposing an intrinsically $\mathrm{X}$-ray weak AGN as a plausible scenario. The relation between $\mathrm{X}$-ray and radio emission can be used to characterize AGNs (Terashima \& Wilson 2003). Using our $5 \mathrm{GHz}$ luminosity upper limit and assuming that most of the hard X-ray emission (1.4 $\times 10^{41} \mathrm{erg} \mathrm{s}^{-1}$ within a region of $\simeq 1.5 \mathrm{kpc}$; Pereira-Santaella et al. 2015b) is due to the AGN, NGC 1614 would be compatible with a LLAGN in Terashima \& Wilson (2003) diagnostic plots. However, according to the $\mathrm{X}$-ray and radio $6 \mathrm{~cm}$ relation by Panessa et al. (2007), the expected $6 \mathrm{~cm}$ luminosity would be $\left(1.1_{-0.8}^{+4.0}\right) \times 10^{37} \mathrm{erg} \mathrm{s}^{-1}$, possibly but unlikely missed by our observations. Anyhow, since most of the hard X-ray luminosity can be explained with SF (Pereira-Santaella et al. 2011), we find it more plausible that NGC 1614 is being essentially powered by stellar activity and/or $\mathrm{SN}$ shocks.

\subsection{The nature of the off-nuclear compact source}

The only significant component detected in our large field of view

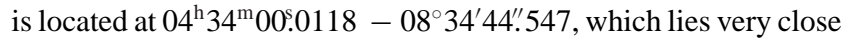
to the centre of region A (see the top panel in Fig. 1), and is located at 0.62 arcsec from the centre of the galaxy, which corresponds to a projected distance of $192 \mathrm{pc}$. We show a zoomed-in map of this 


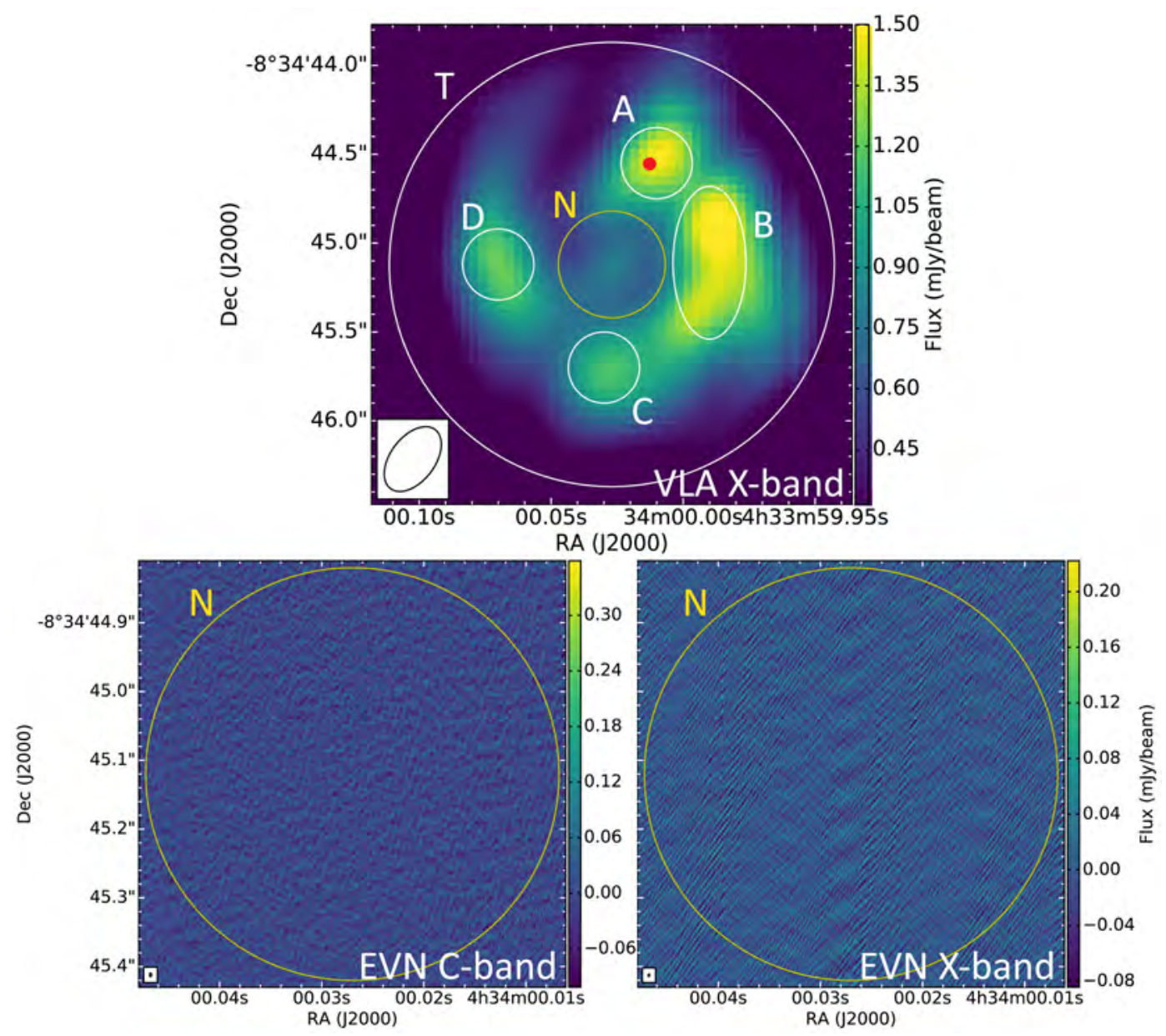

Figure 1. Top panel: VLA $X$-band image of the central kiloparsec region of NGC 1614, with overlaid regions as defined in Herrero-Illana et al. (2014). The synthesized beam size is $0.42 \times 0.25 \operatorname{arcsec}^{2}\left(130 \times 78 \mathrm{pc}^{2}\right)$. The red dot indicates the position of the EVN compact source (see section 3.2). Bottom panels: EVN maps at the $C$ band (left-hand panel) and the $X$ band (right-hand panel) of the nuclear region $\mathrm{N}$, as labelled in the top panel. Synthesized beam sizes are shown in the bottom left-hand panel corners: $5.2 \times 2.8 \mathrm{mas}^{2}\left(1.61 \times 0.87 \mathrm{pc}^{2}\right)$ for the $C$ band and $4.0 \times 1.9 \mathrm{mas}^{2}\left(1.24 \times 0.59 \mathrm{pc}^{2}\right)$ for the $X$ band. The $^{2}$ attained rms are 30 and $28 \mu \mathrm{Jy}_{\text {beam }}^{-1}$, for the $C$ and $X$ bands, respectively.

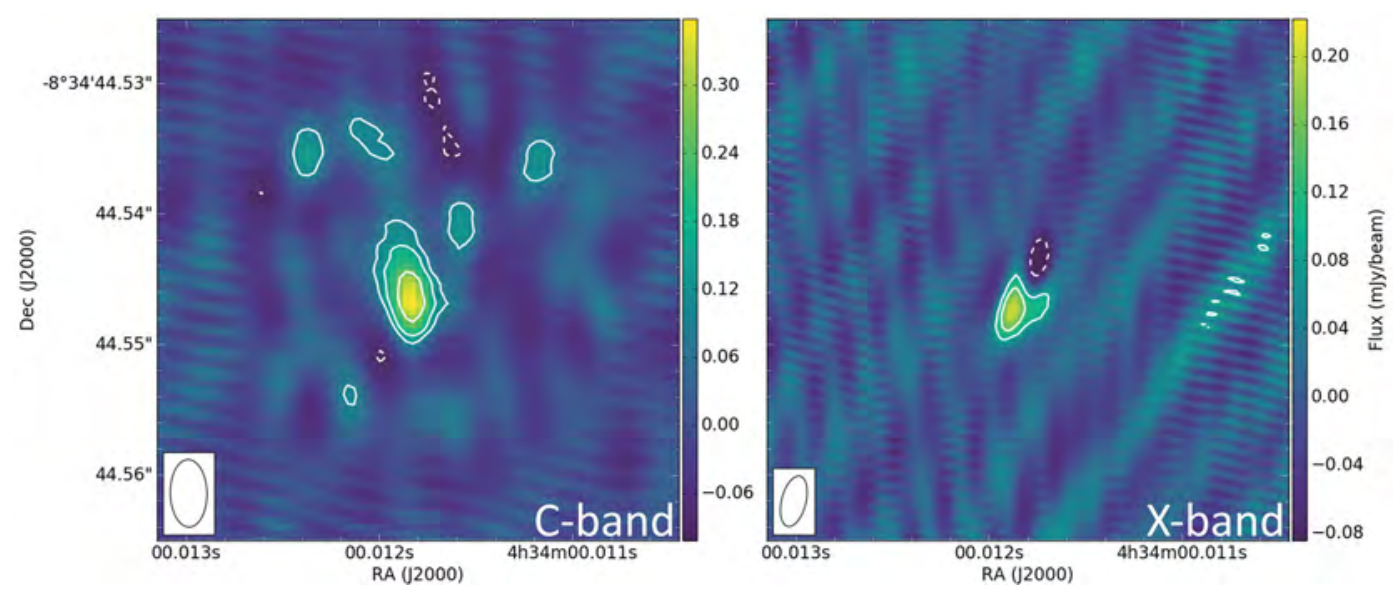

Figure 2. EVN maps of the detected compact source at the $C$ band $(5.0 \mathrm{GHz}$; left-hand panel) and the $X$ band $(8.4 \mathrm{GHz}$; right-hand panel). Contours are plotted at $(-3,3,5$ and 9$)$ times the rms of each image ( 30 and $28 \mu \mathrm{Jy}$ beam $^{-1}$ for the $C$ and $X$ bands, respectively). Negative contours are dashed. The synthesized beam of each image is shown in the bottom left-hand corners: ${ }^{* *} 5.2 \times 2.8 \operatorname{mas}^{2}\left(1.61 \times 0.87 \mathrm{pc}^{2}\right)$ for the $C$ band and $4.0 \times 1.9 \mathrm{mas}^{2}\left(1.24 \times 0.59 \mathrm{pc}^{2}\right)$ for the $X$ band. The peak flux densities are 367 and $215 \mu \mathrm{Jy}_{\text {beam }}{ }^{-1}$ for the $C$ and $X$ bands, respectively. 
area in Fig. 2. The peak flux density, $S_{v}$, of this compact unresolved source is $367 \mu \mathrm{Jy} \mathrm{beam}^{-1}$ at the $C$ band, and $215 \mu \mathrm{Jy} \mathrm{beam}^{-1}$ at the $X$ band. These flux densities correspond to 12 and $8 \sigma$ detections for the $C(5.0 \mathrm{GHz})$ and $X$ bands $(8.4 \mathrm{GHz})$, respectively. We note, however, that the $X$-band data have some sort of phase-calibration problem, as can be seen in the figure, that we were not able to remove completely. The derived two-point spectral index, $\alpha$ (where $\left.S_{v} \propto v^{\alpha}\right)$, is $-1.0 \pm 0.3$. The quoted flux densities, including the systematic uncertainty, correspond to $(9.0 \pm 0.9) \times 10^{36} \mathrm{erg} \mathrm{s}^{-1}$ $(C$ band $)$ and $(8.9 \pm 1.2) \times 10^{37} \mathrm{erg} \mathrm{s}^{-1}(X$ band $)$.

The three most plausible explanations for the nature of this source are (i) an off-nuclear AGN, (ii) a compact super star cluster (SSC), or (iii) a SN or SN remnant. The three possibilities are discussed below:

(i) An AGN is not necessarily located in the innermost nuclear region of a galaxy. On one hand, NGC 1614 is a late-stage merger, with a secondary component that could host an AGN itself. However, the secondary point source revealed in near-IR images $(0.90$ arcsec to the north-east of the main nucleus; Alonso-Herrero et al. 2001; Dametto et al. 2014), and not detected in our EVN observations, is 0.89 arcsec to the east of our detected compact structure. On the other hand, although in some cases the AGN is not located at the centre of the circumnuclear disc (e.g. NGC 1068; García-Burillo et al. 2014), off-nuclear AGNs are often associated with recoiling black holes (Volonteri \& Madau 2008) in dual AGN systems, which is unlikely the case. Finally, AGNs typically exhibit flat radio spectral indices $(\alpha \sim 0)$, unlike our derived index of $\alpha=-1.0 \pm$ 0.3 .

(ii) We can also exclude an SSC nature based on the argument followed by Pérez-Torres et al. (2009) regarding the bright temperature, $T_{\mathrm{B}}$, of the source, which is given by

$T_{\mathrm{B}}=\frac{2 c^{2} B_{v}}{k v^{2}}$,

where $B_{v}$ is the intensity measured in $\mathrm{erg} \mathrm{s}^{-1} \mathrm{~Hz}^{-1} \mathrm{sr}^{-1}$. This depends on the deconvolved size of the source, which we estimate to be $6.1 \times 3.2 \mathrm{mas}^{2}$ for our $C$-band image based on a simple Gaussian fit. We find $T_{\mathrm{B}}=1.25 \times 10^{6} \mathrm{~K}$, an unfeasible value for the thermal emission originated in an SSC. Additionally, the maximum number of ionizing $\mathrm{O}$ stars in such a cluster would produce a thermal luminosity that is still two orders of magnitudes below the observed luminosity (Herrero-Illana, Pérez-Torres \& Alberdi 2012).

(iii) As found in Herrero-Illana et al. (2014) based on the thermal versus non-thermal emission relation, regions A and B (see Fig. 1, top panel) are the most prone to harbour SNe. In particular, region A, where this compact source is located, is a young ( $\sim 8 \mathrm{Myr}$ ), synchrotron-dominated region, likely powered by SNe. This, together with the large $\mathrm{SN}$ rate $\left(0.4 \mathrm{SN} \mathrm{yr}^{-1}\right.$; Herrero-Illana et al. 2014), the short gas depletion time of the ring (Saito et al. 2016), our derived synchrotron spectral index $\alpha=-1.0 \pm 0.3$ and the obtained luminosity, supports the idea that the source has a core-collapse $\mathrm{SN}$ nature. The estimated starburst age of $\sim 8 \mathrm{Myr}$ constrains the initial mass of the progenitor star in the $\simeq 20-30 \mathrm{M}_{\odot}$ range. The detection of $\mathrm{SNe}$ and $\mathrm{SN}$ remnants in enshrouded environments by means of VLBI observations is becoming common with the enhancement in sensitivity of radio interferometers (see e.g. Neff, Ulvestad \& Teng 2004; Pérez-Torres et al. 2009; Ulvestad 2009; Batejat et al. 2011; Bondi et al. 2012; Romero-Cañizales et al. 2012, 2017). While the nature of the core-collapse SN cannot be unambiguously determined without constraining its light curve through a monitoring of the source, the steep spectral index indicates that its radio brightness, $L_{5 \mathrm{GHz}}=(1.8 \pm 0.2) \times 10^{27} \mathrm{erg} \mathrm{s}^{-1} \mathrm{~Hz}^{-1}$, is already diminishing. Furthermore, its high luminosity likely rules out a Type IIP SN (Chevalier, Fransson \& Nymark 2006), which is the most abundant type in the local Universe $(\simeq 59$ per cent of all SNe; Smartt et al. 2009).

The number of SNe found in LIRGs by means of VLBI observations, considering the very few studies available, is uneven. An interesting case is Arp 299, as its IR luminosity $\left(6.7 \times 10^{11} \mathrm{~L}_{\odot}\right)$ and distance (45 Mpc) make it comparable to NGC 1614. From the dozens of compact sources detected in Arp 299 (Pérez-Torres et al. 2009; Bondi et al. 2012), only eight are above our $5 \sigma$ detection threshold $\left(7.4 \times 10^{26} \mathrm{erg} \mathrm{s}^{-1} \mathrm{~Hz}^{-1}\right)$ and have a $\mathrm{SN}$ origin. Furthermore, the SN rate obtained through consistent SED multiwavelength modelling is higher for Arp $299\left(\simeq 0.8 \mathrm{SN} \mathrm{yr}^{-1}\right.$; Mattila et al. 2012) than for NGC 1614 ( $\simeq 0.4 \mathrm{SN} \mathrm{yr}^{-1}$; HerreroIllana et al. 2014). Therefore, we would expect four SN detections in our observations. While the low number statistics may explain this discrepancy, differences in the SF history (Pereira-Santaella et al. 2015a), in the environment (Klessen, Spaans \& Jappsen 2007) or even in the initial mass function (Pérez-Torres et al. 2009) cannot be ruled out. In this sense, we are currently analysing VLBI data on a sample of 10 LIRGs (Pérez-Torres et al., in preparation) for which we have studied their starburst and AGN properties, as well as their SF histories using different diagnostics (Herrero-Illana et al. 2017).

The scenario of a powerful SF ring surrounding a non-active nucleus can be explained by a balance between the in-falling and out-falling gas or by the molecular gas exhaustion in the ring due to the intense SF (Pérez-Torres et al. 2010; Saito et al. 2017), preventing the supermassive black hole from being fed. This is in agreement with the SF-powered molecular outflow found in NGC 1614 by García-Burillo et al. (2015). It is also possible that a massive inflow of gas caused by the final phase of the merging event has not yet been fully funnelled to the central region, which will eventually cause an enhancement in SF, and an active phase of the AGN (König et al. 2016). In any case, our finding supports the model where mechanical heating from SN shocks and superwinds can heat the surrounding medium, inhibiting the accretion of material towards the central AGN and powering the warm gas in the starburst ring of NCG 1614 (Saito et al. 2017).

\section{SUMMARY}

We have obtained radio VLBI observations of the central kiloparsec region of NGC 1614 with the aim of detecting and characterizing a putative AGN and any other compact structure in the circumnuclear ring.

We do not detect any compact structure in the innermost $200 \mathrm{pc}$ region. We have established a tight $5 \sigma$ upper limit luminosity for any putative AGN in this region of $3.7 \times 10^{36}-5.8 \times 10^{36} \mathrm{erg} \mathrm{s}^{-1}$ at 5.0 and $8.4 \mathrm{GHz}$, respectively, ruling out any significant contribution in radio wavelengths. Furthermore, we estimate an AGN contribution of $\lesssim 0.1$ percent in the IR. There is also no hint of any compact radio source in the proposed secondary nucleus of the system.

We detect a compact source in the circumnuclear ring of NGC 1614 located $\simeq 0.6 \operatorname{arcsec}(\simeq 190 \mathrm{pc})$ to the north of the nucleus. Its luminosity $\left(9.0 \times 10^{36}\right.$ and $8.9 \times 10^{36} \mathrm{erg} \mathrm{s}^{-1}$ at the $C$ and $X$ bands, respectively) and spectral shape $(\alpha \simeq-1)$ are indicative of a $S N$ origin, possibly a Type IIn or Ic. Its location in one of the knots of SF of the circumnuclear ring points towards the same direction. This 
supports models using mechanical heating to warm the observed molecular gas in the ring.

In summary, while we cannot rule out completely the existence of an (extremely radio faint) AGN in the central $\sim 200 \mathrm{pc}$ of the nucleus in NGC 1614, its contribution to the radio and IR emissions of the galaxy is negligible. This result, together with the compact and diffuse radio emission in its circumnucleear $\sim \mathrm{kpc}$ region, indicates a virtually pure starburst nature for NGC 1614 .

\section{ACKNOWLEDGEMENTS}

The authors thank the anonymous referee for her/his constructive comments and suggestions. RH-I, AA and MAP-T acknowledge support from the Spanish Ministerio de Economía y Competitividad (MINECO) through grant AYA2015-63939-C2-1-P, co-funded with FEDER funds. AA-H acknowledges support through grant AYA2015-64346-C2-1-P, co-funded with FEDER funds. MP-S acknowledges support from STFC through grant ST/N000919/1, from the John Fell Oxford University Press (OUP) Research Fund, and from the University of Oxford. EVN is a joint facility of independent European, African, Asian and North American radio astronomy institutes. Scientific results from data presented in this publication are derived from the following EVN project code(s): EH030.

\section{REFERENCES}

Alonso-Herrero A., Engelbracht C. W., Rieke M. J., Rieke G. H., Quillen A. C., 2001, ApJ, 546, 952

Alonso-Herrero A., Pereira-Santaella M., Rieke G. H., Rigopoulou D., 2012, ApJ, 744, 2

Batejat F., Conway J. E., Hurley R., Parra R., Diamond P. J., Lonsdale C. J., Lonsdale C. J., 2011, ApJ, 740, 95

Bondi M., Pérez-Torres M. A., Herrero-Illana R., Alberdi A., 2012, A\&A, 539, A134

Chevalier R. A., Fransson C., Nymark T. K., 2006, ApJ, 641, 1029

Condon J. J., 1992, ARA\&A, 30, 575

Dametto N. Z., Riffel R., Pastoriza M. G., Rodríguez-Ardila A., HernandezJimenez J. A., Carvalho E. A., 2014, MNRAS, 443, 1754

García-Burillo S. et al., 2014, A\&A, 567, A125
García-Burillo S. et al., 2015, A\&A, 580, A35

Giroletti M., Panessa F., 2009, ApJ, 706, L260

Herrero-Illana R., Pérez-Torres M. Á., Alberdi A., 2012, A\&A, 540, L5

Herrero-Illana R. et al., 2014, ApJ, 786, 156

Herrero-Illana R. et al., 2017, MNRAS, preprint (arXiv:1705.09663)

Imanishi M., Nakanishi K., 2013, AJ, 146, 47

Klessen R. S., Spaans M., Jappsen A.-K., 2007, MNRAS, 374, L29

König S., Aalto S., Muller S., Gallagher J. S., Beswick R. J., Xu C. K., Evans A., 2016, A\&A, 594, A70

Magnelli B., Elbaz D., Chary R. R., Dickinson M., Le Borgne D., Frayer D. T., Willmer C. N. A., 2009, A\&A, 496, 57

Mattila S. et al., 2012, ApJ, 756, 111

Nagar N. M., Falcke H., Wilson A. S., Ulvestad J. S., 2002, A\&A, 392, 53

Neff S. G., Ulvestad J. S., Teng S. H., 2004, ApJ, 611, 186

Olsson E., Aalto S., Thomasson M., Beswick R., 2010, A\&A, 513, A11

Panessa F., Barcons X., Bassani L., Cappi M., Carrera F. J., Ho L. C., Pellegrini S., 2007, A\&A, 467, 519

Pereira-Santaella M. et al., 2011, A\&A, 535, A93

Pereira-Santaella M. et al., 2015a, MNRAS, 454, 3679

Pereira-Santaella M. et al., 2015b, A\&A, 577, A78

Pérez-Torres M. A., Romero-Cañizales C., Alberdi A., Polatidis A., 2009, A\&A, 507, L17

Pérez-Torres M. A., Alberdi A., Romero-Cañizales C., Bondi M., 2010, A\&A, 519, L5

Risaliti G., Gilli R., Maiolino R., Salvati M., 2000, A\&A, 357, 13

Romero-Cañizales C. et al., 2012, A\&A, 543, A72

Romero-Cañizales C. et al., 2017, MNRAS, 467, 2504

Saito T. et al., 2016, PASJ, 68, 20

Saito T. et al., 2017, ApJ, 835, 174

Sikora M., Stawarz Ł., Lasota J.-P., 2007, ApJ, 658, 815

Smartt S. J., Eldridge J. J., Crockett R. M., Maund J. R., 2009, MNRAS, 395,1409

Terashima Y., Wilson A. S., 2003, ApJ, 583, 145

Ulvestad J. S., 2009, AJ, 138, 1529

Volonteri M., Madau P., 2008, ApJ, 687, L57

Wilson C. D. et al., 2008, ApJS, 178, 189

Xu C. K. et al., 2015, ApJ, 799, 11

This paper has been typeset from a $\mathrm{T}_{\mathrm{E}} \mathrm{X} / \mathrm{LAT}_{\mathrm{E}} \mathrm{X}$ file prepared by the author. 